\title{
Estado reciente de los derechos sexuales y reproductivos de mujeres migrantes que residen en la Ciudad de Buenos Aires
}

\section{Recent Status of Sexual and Reproductive Rights of Migrant Women Residing in the City of Buenos Aires}

\author{
Javiera Verónica Fanta Garrido ${ }^{1}$
}

\section{RESUMEN}

Los derechos sexuales y reproductivos de mujeres migrantes constituyen un tema de interés creciente debido a las dificultades de acceso a los servicios y a suplementos de salud sexual y reproductiva, sumado esto a los obstáculos propios de las instituciones sanitarias. Este trabajo analiza el estado de algunos derechos sexuales y reproductivos de inmigrantes provenientes de países limítrofes y del Perú que residen en la Ciudad de Buenos Aires, Argentina. Mediante datos secundarios y entrevistas a informantes clave, se examina la reproducción adolescente, el acceso a los cuidados preventivos y las características que priman en la atención a la salud sexual y reproductiva de este grupo poblacional.

Palabras clave: 1. migración internacional, 2. género, 3. derechos sexuales; 4. salud sexual y reproductiva; 5. Ciudad de Buenos Aires.

\section{ABSTRACT}

Sexual and reproductive rights of migrant women are a subject of growing interest due to the difficulties in accessing sexual and reproductive health services and supplies, along with constraints inherent to the health care system. This article analyzes the status of some sexual and reproductive rights of immigrant women from neighboring countries and Peru residing in the city of Buenos Aires, Argentina. Using secondary data and interviews with key informants, this research examines adolescent reproduction, access to preventive care, and the predominant characteristics the in sexual and reproductive health care of this population group.

Keywords: 1. international migration, 2. gender, 3. sexual rights, 4. sexual and reproductive health, 5. city of Buenos Aires .

Fecha de recepción: 28 de febrero de 2018

Fecha de aceptación: 15 de mayo de 2019

Publicación en web: 1 de julio de 2020

\footnotetext{
${ }^{1}$ Instituto de Investigaciones Gino Germani-UBA/Conicet, Argentina, javiera.fanta@gmail.com, https://orcid.org/0000-0001-6176-1776
} 


\section{INTRODUCCIÓN}

El dinamismo y la feminización experimentados por los movimientos migratorios a escala global han llevado a situar la Salud Sexual y Reproductiva (SSR) de mujeres migrantes como un tema de atención creciente en la agenda pública y en la literatura especializada. Paralelamente a esto, el propio concepto de SSR se ha ampliado y complejizado en las últimas décadas, al trascender los aspectos meramente reproductivos de la sexualidad y de la idea de ausencia de enfermedad, englobando hoy en día el bienestar físico, mental y social relacionado con la sexualidad y la reproducción (Smith y LeVoy, 2016). En efecto, la definición adoptada por la Conferencia Internacional de Población y Desarrollo en El Cairo (1994), establece que la SSR es "la capacidad de disfrutar de una vida sexual satisfactoria y sin riesgos y de procrear, y la libertad para decidir hacerlo o no hacerlo, cuándo y con qué frecuencia" (Organización de las Naciones Unidas, 1995, p. 37). A partir de su carácter convencional, esta última definición ha dado lugar al establecimiento de una serie de garantías de carácter universal, contenidas en distintos instrumentos internacionales bajo la órbita de los Derechos Sexuales y Reproductivos (DSR).

Este trabajo brinda una aproximación al estudio del estado reciente de algunos DSR de mujeres migrantes provenientes de países limítrofes con Argentina y del Perú, residentes en la Ciudad Autónoma de Buenos Aires (CABA). Específicamente, se examina la situación de las mujeres de 14 a 49 años nacidas en Bolivia, Paraguay y Perú debido al dinamismo adquirido por estos colectivos en los últimos treinta a cuarenta años.

En virtud del amplio abanico de DSR reconocidos, se optó por clasificar estos derechos como aquellos relacionados con: (i) la reproducción, (ii) la no reproducción (anticoncepción y aborto) y (iii) el libre ejercicio de la sexualidad (Monte y Gavernet, 2012). Este trabajo aborda las dos primeras dimensiones a partir de un enfoque metodológico plural. Se analizan los niveles de la fecundidad y maternidad adolescente de las migrantes, sobre el entendido de que este componente representa una manifestación de las desigualdades reproductivas; se examinan las características que priman en el sistema de SSR y que dificultan o actúan como facilitadores para su acceso. Asimismo, se evalúa el acceso efectivo a los cuidados preventivos en materia de SSR de la mujer en el período reciente, y se realiza una breve exposición acerca de la atención de partos recibida por las mujeres involucradas. Para la consecución de estos objetivos se consideró el uso de fuentes de datos secundarios y entrevistas en profundidad. En el primer caso, fueron analizados el Censo Nacional de Población, Hogares y Vivienda 2010 (INDEC, 2015) y la Encuesta sobre Migración, Fecundidad y Familia (EMFF) (Dirección Nacional de Población, 2011), efectuada sobre una muestra no probabilística de migrantes concurrentes a la Dirección Nacional de Migraciones.

Por su parte, las entrevistas fueron realizadas a cuatro informantes clave durante diciembre de 2015 y enero de 2016, entre quienes se encuentran una profesional del Grupo 
de Salud y Migración de la Dirección de Capacitación y Docencia del Ministerio de Salud de la CABA, la coordinadora de la Comisión Argentina para los Refugiados y Migrantes (CAREF) y responsable del proyecto "Acceso a la SSR de mujeres y jóvenes migrantes bolivianos/as", una licenciada en obstetricia, perteneciente al equipo de Parto Respetado de un Hospital Público de la CABA, y una representante del Observatorio de Violencia Obstétrica (Argentina). Por otro lado, este trabajo incorporó seis entrevistas semiestructuradas a mujeres migrantes en edad reproductiva ( 15 a 49 años), nacidas en los respectivos países de estudio y residentes en la CABA, que hubieran tenido hijos nacidos vivos en Argentina, a fin de indagar sobre sus experiencias de parto en el contexto local.

La relevancia de estudiar los DSR de la población femenina migrante se sustenta en varios motivos. Por un lado, las niñas y las mujeres migrantes están más expuestas a sufrir violencia sexual, especialmente en la fase de tránsito (Naciones Unidas, 2016). En efecto, la evidencia muestra que en el contexto de ciertos circuitos migratorios -como es el caso de la migración centroamericana en tránsito por México hacia Estados Unidos-, el riesgo de violación es aceptado y asumido como parte del trayecto (Infante, Silván, Caballero y Campero, 2013).

Adicionalmente, la migración está asociada con un mayor riesgo de contraer infecciones de transmisión sexual (ITS) debido al mayor nivel de exposición de la población móvil a relaciones sexuales forzadas, ocasionales y no protegidas (ONUSIDA, 2013). En este sentido, las mujeres enfrentan un reto adicional, al cargar con el eventual temor a la estigmatización o con el miedo y la vergüenza de haber sido víctimas de violencia sexual, factores que pueden obstaculizar y dilatar la concurrencia a los servicios de salud en caso de haber contraído alguna ITS (Agu, Lobo, Crawford y Chigwada, 2016). Algunos antecedentes referidos a la salud materno-infantil (Haour-Knipe y Grondin, 2003; Carballo, 2006; Heaman et al., 2013; Chiavarini et al., 2016) muestran que las migrantes son menos propensas a realizar controles pre y postnatales y, en general, tardan más tiempo en acudir a la primera consulta para el seguimiento de la gestación. Por otro lado, dado que América Latina y el Caribe es la región del mundo donde se produce la mayor cantidad de abortos inseguros (Sedgh et al., 2016), cabe suponer que las mujeres migrantes sean más proclives a evitar o postergar la asistencia sanitaria ante posibles complicaciones. Todas las circunstancias descritas se profundizan en condiciones de irregularidad migratoria y en migrantes recientes (PNUD, 2008), y si bien es cierto que las características varían de acuerdo a cada grupo migratorio y a las sociedades de destino, existe consenso en que las migrantes merecen una especial atención a la hora de analizar el estado de los DSR de la población. 


\section{CARACTERIZACIÓN DE LA MIGRACIÓN PROVENIENTE DE PAÍSES LIMÍTROFES CON ARGENTINA Y DEL PERÚ}

En rigor, la migración considerada en este texto como limítrofe alude a aquellos colectivos de inmigrados cuyos países de origen comparten frontera con Argentina. Sin embargo, hoy en día el término "limítrofe" implica, más que una referencia geoespacial, una serie de características sociodemográficas que comparten estos migrantes, que los ubica como un grupo social específico. Se trata de una migración racializada (Magliano, 2015) -esto significa que el factor étnico-racial es un enclave que determina la posición de este grupo en la estructura social-, sobrerrepresentada en ciertos nichos laborales, sujeta a condiciones laborales precarias e irregulares, e impulsada por proyectos de subsistencia y movilidad social. En virtud de estos rasgos, la literatura especializada suele englobar dentro del concepto de migración limítrofe a las personas nacidas en Perú, pese a ser un país que no comparte frontera geográfica con Argentina (Cerrutti, 2009).

Según datos del Censo Nacional de Población, Hogares y Viviendas 2010, en la CABA residen 90000 mujeres en edad reproductiva nacidas en Bolivia, Paraguay y Perú (Instituto Nacional de Estadística y Censos, 2010). Si bien las razones de la migración son variadas, la búsqueda de alternativas laborales predomina como motor de desplazamiento entre las migrantes limítrofes (Cerrutti, 2009). Su inserción en el mercado de trabajo se concentra en unas cuantas ocupaciones, destacándose el servicio doméstico como la principal actividad y, en menor medida, el comercio minorista y la actividad agrícola (Íbid.).

Con respecto a los antecedentes sobre la atención de estos colectivos migratorios de la salud en general, se advierte que la construcción de estereotipos en torno a su origen actúa como limitante de acceso y permanencia en el sistema. Jelin, Grimson y Zamberlin (2006) observaron que los médicos de instituciones de salud en la capital del país y los 24 partidos que componen el área del Gran Buenos Aires (GBA), ${ }^{2}$ distinguen entre las migrantes paraguayas y peruanas, por un lado, y el colectivo boliviano por otro, en función de su nivel de adaptabilidad a la cultura local. En este último caso, los profesionales y administradores de la salud identifican una brecha cultural más amplia, que es atribuida al menor nivel educativo promedio de las migrantes bolivianas, lo que estaría expresado en el plano de la interpretación de consignas y el seguimiento de indicaciones médicas. Los autores sostienen que en el caso del sistema hospitalario, esta brecha deriva en procesos de inferiorización del otro, mientras que en los sistemas de atención primaria las diferencias culturales suelen ser relativizadas e identificadas como un problema de comunicación.

Por su parte, el trabajo de Cerrutti (2010) referido a mujeres bolivianas en la zona de la frontera, en Provincia de Buenos Aires y en la CABA, reveló que los profesionales y

${ }^{2}$ El GBA es el gran conjunto urbano comprendido por la CABA más los partidos del conurbano bonaerense, correspondientes estos a 24 jurisdicciones administrativas. 
administradores de la salud concuerdan en señalar que las migrantes de este origen muestran una escasa autonomía en las decisiones reproductivas, y que poseen poca predisposición a realizarse controles y seguimientos; esto ocurre, por ejemplo, con los controles prenatales recomendados.

Wang (2010), en su exhaustiva investigación sobre migrantes paraguayas en el GBA, advierte que en ciertos sectores del Paraguay resulta innecesario y hasta inútil acudir a instituciones de salud, si no hay una enfermedad que justifique la consulta. Más aún, muchas de estas migrantes toman contacto con el sistema recién al momento de parir, y suele estar acotado a ese acontecimiento, interrumpiéndose una vez finalizado el proceso de parto y posparto inmediato. Con referencia a las prácticas contraceptivas, la autora advierte que la migración representa un hito en la vida de estas mujeres, al facilitar el acceso a los métodos anticonceptivos (MAC) y promover las consultas en los servicios de SSR.

\section{Normativa argentina para la protección de los derechos sexuales y reproductivos}

En 2003, dos años después de ocurrida una de las crisis económicas más profundas que azotara Argentina, el país dio inicio una nueva etapa en términos de modelo social, caracterizada por el despliegue de acciones desde el Estado tendientes al fortalecimiento de los procesos de inclusión. Bajo esa óptica, el Congreso Nacional promulgó en 2004 la ley $\mathrm{N}^{\mathrm{o}} 25.871$ de Migraciones, mediante la cual se garantiza el derecho a la libre circulación de personas en todo el territorio nacional. Particularmente en relación al derecho a la salud, la ley establece en su artículo 8 que no podrá negársele o restringírsele el derecho a la salud (incluyendo la asistencia social y la atención sanitaria) a ningún extranjero que lo requiera, cualquiera que sea su estatus migratorio. Más aún, delega en las autoridades de los establecimientos sanitarios, así como también de las instituciones educativas, la responsabilidad de brindar orientación y asesoramiento sobre los trámites correspondientes para subsanar la irregularidad migratoria.

En consonancia con este enfoque político de mayor vigilancia y respeto por los derechos fundamentales de las personas, el año 2002 se sancionó la ley $\mathrm{N}^{\circ} 25.673$, que crea el Programa Nacional de Salud Sexual y Procreación Responsable (PNSSyPR) bajo la órbita del Ministerio de Salud. Este programa está destinado a la población en general, sin discriminación alguna, y comprende fines fundamentalmente preventivos. Entre sus objetivos se encuentran la disminución de la morbimortalidad materno-infantil, la prevención de embarazos no deseados, y la prevención y detección precoz de enfermedades de transmisión sexual, de VIH/SIDA y de patologías genitales y mamarias (artículo $2^{\circ}$ de la ley).

Adicionalmente, en Argentina existe la ley $\mathrm{N}^{\circ} 25.929$ sobre Parto Respetado, sancionada en 2004 y reglamentada en 2015 a través del decreto 2035/15. Esta normativa protege los derechos de la madre y del bebé en la etapa de nacimiento, y los derechos de la mujer en 
relación con el embarazo. Entre ellos, contempla el derecho al trato digno y respetuoso de las pautas culturales, el derecho a ser informada sobre la evolución del parto y las posibles intervenciones que pudiesen tener lugar en las distintas etapas del proceso, y la protección del vínculo corporal entre la madre y el/la recién nacido/a (artículo 2 del decreto reglamentario).

Uno de los aspectos más novedosos de esta legislación es que aborda un tipo de violencia de género típicamente invisibilizada: la violencia obstétrica, entendida como aquella que es ejercida por el personal de salud sobre el cuerpo y los procesos reproductivos de los cuerpos gestantes. Si bien esta modalidad de violencia está contemplada por la ley $\mathrm{N}^{\circ} 26.485$ de protección integral a las mujeres, la ley de parto respetado establece garantías específicas, referidas a la autonomía de voluntad y al respeto por la dignidad humana en los procesos de gestación y nacimiento. Otro aspecto relevante es que incorpora una mirada flexible acerca de la forma en que pueden ser abordados los procesos de gestación y parto. Por ejemplo, el artículo 2 del decreto reglamentario establece que cada persona tiene derecho a elegir el lugar y la forma en la que va a transitar su trabajo de parto, así como también la vía de nacimiento. Además, el artículo 2 señala que en relación con el embarazo, el trabajo de parto, el parto y el puerperio, toda mujer tiene derecho a ser tratada con respeto y dignidad, y a no ser discriminada por su cultura, etnia, preferencias, ni factores de otra índole. Desde una óptica intercultural, esta ley pone en cuestión el uso de protocolos estandarizados para la atención de partos sin riesgo, y provee un marco normativo lo suficientemente amplio para incorporar una mirada dinámica de los procesos culturales asociados a la gestación y al parto.

\section{La reproducción adolescente de migrantes limitrofes en la Ciudad de Buenos Aires}

La evaluación de la reproducción adolescente es un ejercicio no exento de dificultades, especialmente cuando el análisis abarca a la población migrante. En primer lugar, existe el reconocido problema de la omisión en la declaración de nacimientos a niñas y adolescentes, posiblemente atribuidos a las connotaciones negativas que encierra el embarazo y la maternidad en edades tempranas. Por otro lado, la evaluación de este componente en mujeres migrantes plantea la dificultad de distinguir el lugar en que se produjo el embarazo y el nacimiento de los hijos. Además, existen diferencias respecto de los nacimientos acontecidos en estadios precoces (14 años) y tempranos de la adolescencia (15-17 años), y aquellos que ocurren en edades avanzadas (18-19 años), tanto por factores fisiológicos como contextuales. En este sentido, la incidencia de la morbimortalidad materna en edades tempranas es comparativamente mayor a la que se produce en edades tardías y, en determinados contextos, ser madre a partir de los 17 años puede resultar un hecho socialmente aceptable o deseable. Atendiendo a esta última consideración, se analizó la maternidad en edades precoces, tempranas y avanzadas de la adolescencia -asumiendo que un nacimiento antes de los 18 años suele ser un evento no deseado, independientemente del 
país de origen- y por otro lado, la tasa de fecundidad para el conjunto de adolescentes de 15 a 19 años.

Los resultados muestran que el conjunto de migrantes limítrofes y del Perú expresa porcentajes marcadamente superiores de maternidad adolescente (14 a19 años) respecto de la población nativa (cuadro 1). Los diferenciales registrados en las edades precoces y tempranas de la adolescencia son especialmente pronunciados, sobre todo con respecto a la colectividad boliviana y paraguaya. En consonancia con estos resultados, las tasas específicas de fecundidad de las migrantes limítrofes de 15 a 19 años presentan diferencias notorias según país de nacimiento, excediendo, en los distintos casos, al nivel expresado por las nativas argentinas (cuadro 2).

Cuadro 1. Porcentaje de madres adolescentes precoces (14 años), tempranas (15-17 años) y avanzadas (18-19 años), según país de nacimiento. Ciudad Autónoma de Buenos Aires, 2010

\begin{tabular}{lccc}
\hline País de nacimiento & \multicolumn{3}{c}{ Madres adolescentes (\%) } \\
& 14 años & $15-17$ años & $18-19$ años \\
\hline Argentina & 1.6 & 7.2 & 21.6 \\
Bolivia & 4.7 & 13.1 & 31.7 \\
Paraguay & 4.0 & 10.8 & 28.3 \\
Perú & 4.4 & 8.6 & 19.2 \\
Total migrantes limítrofes y del Perú & 4.3 & 11.2 & 27.9 \\
\hline
\end{tabular}

Fuente: Censo Nacional de Población, Hogares y Vivienda 2010. Cuestionario Ampliado (Instituto Nacional de Estadística y Censos, 2010).

Cuadro 2. Tasa Específica de Fecundidad (TEF) 15 a 19 años, según país de nacimiento. Migrantes limítrofes y del Perú y nativas argentinas. Ciudad Autónoma de Buenos Aires, 2010

\begin{tabular}{|c|c|}
\hline País de nacimiento & TEF $_{15-19}$ (por mil) \\
\hline Argentina & 26.5 \\
\hline Bolivia & 81.2 \\
\hline Paraguay & 86.9 \\
\hline Perú & 64.1 \\
\hline Total migrantes limítrofes y del Perú & 77.9 \\
\hline
\end{tabular}

Fuente: Censo Nacional de Población, Hogares y Vivienda 2010.

Cuestionario Ampliado (Instituto Nacional de Estadística y Censos, 2015)

Cabe pensar que los resultados expuestos no son la manifestación de una sana heterogeneidad cultural, sino que representan brechas inquietantes que estarían reflejando un acceso desigual a recursos y derechos (entre ellos, a una vida sin violencia y a tomar decisiones sobre el propio cuerpo, por nombrar solo algunos). 


\section{Acceso a métodos anticonceptivos}

Como se ha señalado anteriormente, el análisis sobre el uso de MAC y su vínculo con el derecho que tienen las mujeres a decidir sobre sus procesos reproductivos debería considerar un aspecto fundamental desde el punto de vista de la autonomía del cuerpo: esto es, la voluntad que efectivamente ellas tienen de hacer uso de estos métodos o, en su defecto, expresar el deseo de postergar la maternidad, o bien de concluir/impedir la reproducción.

Como primer paso, este apartado presenta una caracterización de la muestra analizada por la EMFF, con énfasis en las mujeres migrantes residentes en la CABA. A continuación, se compara el número de hijos nacidos vivos con el número ideal de hijos declarado, con el propósito de conocer en qué medida la trayectoria de la fecundidad coincide con las aspiraciones reproductivas de las encuestadas. Para obtener esta última información, la EMFF implementó la pregunta "¿Cuál cree usted que es el número ideal de hijos que una mujer debiera tener?". Al respecto, se debe advertir que puede haber una tendencia a declarar una cantidad no inferior al número efectivo de hijos vivos y que, en ciertos contextos, pueden aparecer respuestas no cuantificables (por ejemplo, "lo que dios quiera"). Tomando en cuenta lo anterior, así como la influencia que ejercen las variables de mortalidad infantil y mortalidad a lo largo de la vida, se descartaron los casos no cuantificables y aquellos que declaraban la muerte de algún hijo, respectivamente. Finalmente, se analizó el uso actual de MAC por parte de migrantes que no desean tener más hijos, o que desean postergar un próximo o primer embarazo.

La muestra analizada se compone de 451 migrantes, 171 de ellas nacidas en Bolivia, 173 en Paraguay y 107 procedentes de Perú. La edad de las encuestadas varía en el rango de 18 a 49 años, con una edad media de 32 años. Aproximadamente la mitad de estas mujeres (48\%) llegó al país entre los 20 y 29 años, y solo una proporción menor (16\%) migró después de los 30 años. Cerca de las tres cuartas partes reside en Argentina desde hace 5 años o más (296 mujeres), de manera que se trata de una muestra compuesta principalmente por migrantes antiguas. Al momento de la encuesta la mayoría asistió o asistía al sistema de educación formal, siendo el colectivo paraguayo el que presenta el mayor nivel educativo (4.6 años promedio), seguido de las migrantes peruanas y bolivianas, cuyo nivel promedio alcanza los 3.9 años de escolaridad, respectivamente. En relación al estado conyugal, 70 por ciento de las encuestadas (318 migrantes) se encuentra actualmente en pareja, de las que más de la mitad habita con su cónyuge ( 274 mujeres). Además, cerca de 70 por ciento (317 mujeres) es o ha sido madre alguna vez.

Al analizar el número ideal de hijos reportado por las migrantes de países limítrofes, los datos muestran que la aspiración predominante, indistintamente del origen migratorio, es el modelo de los 2 hijos (55\%). Esta preferencia es declarada principalmente por mujeres que al momento del relevamiento, aún no habían iniciado su trayectoria reproductiva. Destaca el hecho de que ninguna de las encuestadas aspira a la nuliparidad. En relación con la cantidad 
de hijos nacidos vivos por mujer, se observa que el promedio varía entre 1.4 entre las migrantes peruanas y 1.8 en las bolivianas, mientras que las migrantes paraguayas presentan una media de 1.5 hijos. Es probable que estos resultados, que expresan una baja intensidad de la fecundidad, se deban a que la muestra abarca esencialmente a mujeres jóvenes que aún no han concluido su etapa reproductiva.

Con el fin de comparar las aspiraciones reproductivas con la trayectoria de la fecundidad de las migrantes encuestadas, el cuadro 3 ilustra el número ideal de hijos declarado según la cantidad de hijos nacidos vivos, expresado en porcentaje (\%). De allí se desprende que la planificación familiar presentaría mayores deficiencias entre quienes poseen una mayor cantidad de hijos. Si tomamos como referencia a las migrantes que tuvieron 4 o más hijos, por ejemplo, observamos que solo una pequeña parte de ese grupo considera que esa es una cantidad ideal.

Cuadro 3. Migrantes limítrofes y del Perú (18-49 años) por número ideal de hijos, según cantidad de hijos nacidos vivos (HNV) $(\%)$

\begin{tabular}{|c|c|c|c|c|c|c|}
\hline \multirow{2}{*}{$\mathrm{HNV}$} & \multicolumn{4}{|c|}{ Número ideal de hijos (\%) } & \multirow{2}{*}{ Total } & \multirow[t]{2}{*}{$N$} \\
\hline & 1 & 2 & 3 & $4+$ & & \\
\hline 0 & 3 & 62 & 27 & 8 & 100 & 133 \\
\hline 1 & 8 & 60 & 24 & 8 & 100 & 108 \\
\hline 2 & 3 & 59 & 28 & 10 & 100 & 96 \\
\hline 3 & - & 29 & 50 & 21 & 100 & 66 \\
\hline $4+$ & - & 57 & 34 & 9 & 100 & 44 \\
\hline
\end{tabular}

Fuente: Encuesta sobre Migración, Fecundidad y Familia 2011 (Dirección Nacional de Población, 2011).

Ahora bien, ¿qué ocurre con aquellas migrantes que completaron sus aspiraciones reproductivas? Las mujeres de origen limítrofe que se encuentran actualmente en pareja, ¿hacen uso de MAC para prevenir un eventual embarazo? La información analizada revela que del total de migrantes unidas, un 40 por ciento (128 mujeres) completó sus preferencias reproductivas; es decir, el número de hijos tenidos iguala o supera al número ideal de hijos declarado. De ellas, 65 por ciento hace uso de algún MAC (86 mujeres), mientras que un 32 por ciento (41 encuestadas) no utiliza actualmente ningún método para prevenir embarazos, pese a haber alcanzado la cantidad que considera ideal. En línea con estos resultados, los datos muestran que ante la pregunta “¿le gustaría tener más hijos?”, 34 por ciento de las migrantes que convive en pareja y que desean finalizar su ciclo reproductivo (50 casos) no hace uso de ningún método para evitar embarazos.

Lo anterior conduce a pensar que una proporción no menor de las migrantes encuestadas carece de acceso pleno a los servicios de planificación familiar, o bien, no posee información acerca del modo de uso de los MAC distribuidos. Incluso en los casos en los que la razón para no utilizar MAC por parte de mujeres que desean finalizar su carrera reproductiva, o 
postergar un embarazo, apunta al temor de eventuales efectos secundarios, lo que estaría fallando sería el acceso a la información y a opciones alternativas de MAC.

\section{Cuidados preventivos en salud reproductiva}

Las mujeres viven en promedio de seis a ocho años más que los hombres y sin embargo, esa diferencia no es indicativa de que sus necesidades sanitarias estén siendo efectivamente cubiertas. La propia Organización Mundial de la Salud (OMS) afirma que la función de la desigualdad entre los sexos en el aumento de la exposición a los riesgos y la vulnerabilidad frente a estos, limita el acceso a la asistencia e información sanitarias, repercutiendo en detrimento de los resultados de la salud de las mujeres (OMS, 2009).

Asimismo, en lo que respecta a la cobertura de los servicios de salud, el organismo internacional reconoce que es más probable que se disponga de ciertos servicios sanitarios, como la atención durante el embarazo, que de otros, como el tratamiento del cáncer cervicouterino. Este tipo de cáncer, específicamente, es el más frecuente después del cáncer de mama en las mujeres de las regiones en desarrollo y de Argentina en particular. En el país, la tasa de mortalidad por cáncer de cuello de útero alcanza los 8.3 casos por cada 100 000 mujeres, un equivalente a 1800 muertes anuales, mientras que el cáncer mamario es responsable de 5600 defunciones por año a nivel nacional (International Agency for Research on Cancer, 2012).

El cáncer cervicouterino, puntualmente, refleja la fuerte desigualdad social que prevalece en el ámbito de la salud de la mujer, ya que se trata de una enfermedad casi completamente prevenible gracias a los conocimientos y tecnologías actualmente disponibles. El Ministerio de Salud de la Nación ha reconocido que se trata de un tipo de enfermedad que afecta principalmente a mujeres de bajos recursos socioeconómicos que, por diferentes barreras sociales, no acceden a los servicios de tamizaje (Zamberlin, Thouyaret y Arrossi, 2011). En virtud de lo anterior, las pruebas de detección resultan fundamentales para alertar sobre la existencia de lesiones precancerosas o cancerosas, y revertir así el nivel de mortalidad por estas causas. La mamografía y la citología convencional (Papanicolau o más comúnmente denominado PAP) son las pruebas que en rigor se utilizan para detectar el cáncer mamario y de cuello de útero, respectivamente.

Ahora bien, ¿en qué medida las migrantes limítrofes en edad reproductiva acuden a los servicios de ginecología? Al respecto, la literatura especializada muestra que la concurrencia a los servicios de salud en general, y de SSR en particular, está estrechamente relacionada con la antigüedad de la residencia en el destino (Chen et al., 2001; Carballo, 2006); es decir, que la probabilidad de asistencia a los servicios sanitarios se incrementa en la medida que aumenta la cantidad de años de residencia. Considerando este antecedente, el cuadro 4 muestra el porcentaje de migrantes limítrofes que ha realizado al menos una consulta ginecológica en Argentina, según el tiempo agrupado en años de residencia en el país. Los 
resultados muestran que más de tres cuartas partes de estas mujeres asistió alguna vez al ginecólogo $(86 \%)$ y refuerzan la idea de que existiría una relación entre la antigüedad de la migración y la concurrencia a este tipo de servicio sanitario. En efecto, entre las migrantes que llevan viviendo más de 10 años en el país, solo una pequeña parte no consultó nunca un ginecólogo (6\%), mientras que entre las mujeres que llevan menos de tres años, este porcentaje llega a casi la mitad (46\%). En cambio, los resultados no varían de manera importante si se analizan en función del país de nacimiento.

Cuadro 4. Migrantes limítrofes y del Perú (18-49 años) residentes en CABA que asistieron alguna vez al ginecólogo en Argentina, según tiempo de residencia en el país (\%)

\begin{tabular}{lcccc}
\hline \multirow{2}{*}{$\begin{array}{l}\text { Tiempo de residencia en } \\
\text { Argentina }\end{array}$} & \multicolumn{3}{c}{ Asistió al ginecólogo en } & \multirow{2}{*}{ Argentina (\%) } \\
& Sí & No & Total & \\
\hline Menos de 3 años & 54 & 46 & 100 & 50 \\
3 a 5 años & 81 & 19 & 100 & 104 \\
6 a 10 años & 90 & 10 & 100 & 100 \\
Más de 10 años & 94 & 6 & 100 & 196 \\
Total & 86 & 14 & 100 & 450 \\
\hline
\end{tabular}

Fuente: Encuesta sobre Migración, Fecundidad y Familia 2011 (Dirección Nacional de Población, 2011)

En el cuadro 5 se ilustra el porcentaje de migrantes limítrofes y del Perú de entre 30 y 49 años de edad que se ha realizado una mamografía alguna vez en Argentina y un PAP en el último año, por antigüedad migratoria. Los datos no permiten distinguir la fecha de realización del último control mamario, por lo que solo se incluye la realización del examen al menos una vez durante el tiempo de residencia en Argentina, como medida de aproximación a los cuidados preventivos en SSR de las migrantes.

Los resultados muestran que el porcentaje de encuestadas que se efectuó alguna vez una mamografía en el país se incrementa con la antigüedad de la residencia. La excepción a esta tendencia la constituye el grupo con menor antigüedad migratoria, pero es probable que esto se deba al reducido número de casos que abarca el grupo de migrantes más recientes. Con independencia de esta variación, llama la atención que en promedio más de la mitad de las migrantes no se ha sometido a esta prueba durante su residencia en el país, pese a las recomendaciones médicas establecidas para las mujeres de este rango etario. La frecuencia relativa del PAP en el último año, en cambio, es significativamente mayor en comparación a este último indicador. En promedio, un 68 por ciento de las migrantes de entre 30 y 49 años se había realizado un PAP durante el año anterior a la encuesta. Asimismo, los resultados muestran que este porcentaje no aumenta con la antigüedad migratoria, como sí ocurre en el caso anterior. 
Cuadro 5. Migrantes limítrofes y del Perú (30-49 años) residentes en CABA que se han hecho una mamografía en Argentina y PAP en el último año, según tiempo de residencia en el país (\%)

\begin{tabular}{lcccccccc}
\hline \multirow{2}{*}{\begin{tabular}{c} 
Tiempo de residencia en \\
\multicolumn{1}{c}{ Argentina }
\end{tabular}} & \multicolumn{3}{c}{$\begin{array}{c}\text { Se hizo mamografía en } \\
\text { Argentina (\%) }\end{array}$} & \multicolumn{5}{c}{ Se hizo un PAP en el último } \\
& Sí & No & Total & $N$ & Sí & No & Total & $N$ \\
\hline Menos de 3 años & 40 & 60 & 100 & 15 & 67 & 33 & 100 & 15 \\
3 a 5 años & 35 & 65 & 100 & 29 & 72 & 28 & 100 & 29 \\
6 a 10 años & 38 & 62 & 100 & 48 & 67 & 33 & 100 & 48 \\
Más de 10 años & 47 & 53 & 100 & 151 & 67 & 33 & 100 & 151 \\
Total & 43 & 57 & 100 & 243 & 68 & 32 & 100 & 243 \\
\hline
\end{tabular}

Fuente: Encuesta sobre Migración, Fecundidad y Familia 2011 (Dirección Nacional de Población, 2011)

Otro hallazgo que reflejan los resultados obtenidos, coherente con los antecedentes bibliográficos en esta materia, se relaciona con la baja proporción de migrantes bolivianas que se ha efectuado las pruebas recomendadas para la prevención de cáncer mamario y cérvicouterino, en comparación con las mujeres de los colectivos paraguayo y peruano. Los datos de la EMFF referidos a migrantes de 30 a 49 años de edad residentes en la CABA, muestran que mientras 53 por ciento de las paraguayas (90 mujeres) y 41 por ciento de las peruanas (59 mujeres) se realizaron alguna vez una mamografía en el país, entre las bolivianas esta cifra llega al 35 por ciento (94 mujeres). Análogamente, aunque con diferencias menos marcadas, el porcentaje de migrantes nacidas en Bolivia que se hizo un PAP en el último año alcanza 63 por ciento, en comparación a un 74 y 66 por ciento de las nacidas en Paraguay y Perú, respectivamente.

Los resultados expuestos hasta aquí obligan a pensar la migración como un fenómeno interseccional, según el cual la propia categoría de migración limítrofe resulta acotada para explicar el acceso diferencial a los servicios y cuidados preventivos en SSR. De acuerdo a lo observado, tanto la antigüedad migratoria como el país de origen de las migrantes representarían factores diferenciales de acceso. Considerando que se trata de pruebas preventivas y de controles necesarios para la SSR de toda mujer, junto con el hecho de que constituyen puntos prioritarios para la puesta en marcha efectiva del PNSSyPR, cabe pensar que los grupos con las características señaladas se encuentran más expuestos a las vulnerabilidades en este ámbito de la salud.

Características de la atención a la Salud Sexual y Reproductiva de migrantes limítrofes y del Perú, con énfasis en la atención de partos

Dado que existe consenso sobre el hecho de que la población migrante representa un grupo de interés en el marco de la SSR, cabe preguntarse ¿cómo opera el sistema biomédico en la atención a la salud de mujeres migrantes a partir de la identificación del colectivo migratorio 
limítrofe, en tanto grupo social, y de las prácticas implementadas a partir dicha identificación? (Aizenberg y Maure, 2017) ¿Cómo se concibe y es aplicada la normativa vigente en materia DSR? ¿Cuáles son los principales obstáculos y facilitadores que afectan el cumplimiento efectivo de estos derechos?

Una de las primeras cuestiones que emerge en el marco de los DSR de las migrantes nos remite a la factibilidad y los modos de incorporar una mirada intercultural al sistema de SSR y, más específicamente, a la atención de partos. Desde ese punto de partida nos preguntamos, ¿qué es lo que se entiende por integración de la cultura al sector de SSR y de qué cultura estamos hablando? En relación a esto es posible detectar la presencia dos líneas principales (Comelles, 2004): una que tiende a la comprensión de lo cultural como una taxonomía étnica, y otra que lo aborda como significado, es decir, como una dimensión subjetiva más amplia que, en el caso de la SSR, está asociado al ser mujer, ser trabajadora, ser (o no) indígena, hablar determinado idioma, etcétera, y desde esa base, ser reconocida como sujeta de derechos. Por su parte, lo cultural entendido desde la etnicidad suele ir de la mano con la exotización de lo foráneo, cuyo riesgo es derivar en categorías estereotipadas acerca de lo que significa comunicarse con y atender las necesidades del Otro.

Curiosamente, los que son más sensibles con la cuestión migratoria tienen más dificultades en observar la violencia contra la mujer. En parte a veces tienen esto del relativismo cultural, esa visión culturalista de respetar tanto la cultura del otro y no ver esto, que dicen algunos, [de] que se termina el relativismo cultural cuando hay una violación de derechos [...] dicen 'porque como yo soy blanca, no le quiero meter mis ideas'. [...] Por ejemplo, una profesional que le dice desprejuiciadamente a una mujer 'bueno, puede planificar sus embarazos', y esa mujer quizás tiene 12 hermanos y tuvo un solo hijo, y ese cambio se dio de una generación a otra. Creo que sabemos muy poco acerca de eso [...] ¿Qué le pasó subjetivamente a esa mujer? (Laura, Grupo de Salud y Migraciones del Ministerio de Salud de CABA; comunicación personal, 28 de diciembre de 2015).

Cuando la mirada de los profesionales y administradores de la salud se enfoca en "lo tradicional" o "lo indígena" y se opacan aspectos complementarios que conforman la subjetividad del usuario/a, pueden aparecer dificultades en el servicio de atención, que van desde suprimir información que afecta la optimización de los servicios de SSR - por ejemplo, desconocer cuál es el público objetivo sobre el que es necesario enfatizar los métodos de planificación familiar-, hasta la afectación de derechos y, por extensión, de la dignidad humana -por ejemplo, invisibilizar situaciones de violencia-.

Contrariamente a lo antes descrito, cuando lo cultural es concebido como constitutivo de la subjetividad (como episteme), se generan las condiciones para un intercambio igualitario entre las diferentes sensibilidades culturales (Fernández Juárez, 2011). Esta mirada, propia de la perspectiva intercultural, exige el desmoronamiento del pensamiento monolítico y de la esencialización, del repliegue sobre uno mismo y de la ignorancia del "otro" (Ibíd.), características éstas del sistema biomédico hegemónico. Esta visión es la que intenta incorporar el equipo de Parto Respetado de un Hospital Público de la CABA, a través de la implementación de un modelo de atención de partos en consonancia con la ley $\mathrm{N}^{\circ} 25.929$ : 
Utilizamos tiras, usamos pelotas, tenemos el banquito, la lámpara de sal, esencias, aceites. Ellas [las parturientas] deciden, y nosotros también evaluamos que estén aptas para esto. Pasan su trabajo de parto con un baño, con duchas si quieren, con la persona que ellas elijan. A veces rotan las personas [los acompañantes] porque están cansadas o porque pasan muchas horas. Y después podemos parir en el mismo dilatante, o si la persona prefiere ir a una camilla para que le sea más fácil pujar, o lo que vayan necesitando. Lo más importante que me parece es que puedan elegir y que puedan estar acompañadas. [...] Nosotras tratamos de darle el confort que le podemos generar en un lugar que es público. Lo que prima también es siempre el buen trato, la sonrisa, la mirada [...] Buscá lo que tu cuerpo te pida (Adriana, obstétrica de un Hospital Público de la CABA; comunicación personal, 6 de enero de 2016).

En la implementación de esta iniciativa, la dimensión cultural se plasma en la posibilidad de elegir, esto es, al entender que no todas las mujeres pueden ni desean realizar el trabajo de parto y el parto de una misma manera; pero, sobre todo, se trata de elegir entre opciones que fueron pensadas a partir de la experiencia de atención a distintos cuerpos.

Desde esta perspectiva no se busca omitir al ser migrante, sino más bien se apunta a una articulación de los diferentes aspectos constitutivos de la mujer, de la migrante, de la trabajadora, de la indígena; todos ellos terrenos en los que también actúa la vulneración de derechos.

Esta mirada interseccional resulta especialmente útil a la hora de analizar el fenómeno de la violencia obstétrica en el contexto de los DSR de las mujeres. Entre otros aspectos, este tipo de violencia implica maltrato físico o verbal, la falta de atención o consideración hacia la mujer, intervenciones médicas injustificadas, y la ausencia del pedido de consentimiento informado. Si bien todas las mujeres que atraviesan un proceso de preparto, parto y postparto en instituciones de salud se encuentran expuestas a ella, la condición migratoria determinaría un eje adicional de vulnerabilidad que tiende a reforzar la aparición de relaciones desiguales en este ámbito:

Como migrante te enfrentás a algo completamente desconocido. Vas a experimentar una relación realmente asimétrica en la gran mayoría de los casos y encima sos de otro país. Y si encima tu color de piel y tu fisionomía no se adapta... Desde ya corren con mucha más desventaja que una persona nativa (Marina, Observatorio de Violencia Obstétrica, comunicación personal, 14 de diciembre de 2015).

De esta manera, el género no sería la única dimensión involucrada en la determinación del ejercicio efectivo de los DSR, pues este se encuentra condicionado también por otras categorías intervinientes, como son la migración, la raza, la orientación sexual, entre otras. ¿Desde qué lugar abordar, entonces, esta "vulnerabilidad adicional"? ¿Implica ello segmentar las demandas por el cumplimiento de los DSR? Dado que se trata de un fenómeno transversal, la respuesta de los informantes a estas interrogantes apunta a sostener la construcción de prácticas garantistas no solo desde el ser migrante, sino también desde el 
ser mujer, reconociendo dentro de ese marco global que las situaciones de exclusión, discriminación u opresión afectan mayormente a mujeres atravesadas por ejes adicionales de subordinación.

Con referencia a las características que priman en la atención de la SSR de migrantes, es posible constatar que si bien los informantes entrevistados reconocen la existencia de un modelo hegemónico de atención biomédica, al mismo tiempo identifican grietas $\mathrm{y}$ resistencias que, al manifestarse, hacen de los profesionales de la salud un grupo altamente heterogéneo. Es por esto que no sería correcto hablar de en un único colectivo de profesionales de la salud.

Están los que tienen una sensibilidad especial con el tema, gente que viene trabajando hace muchísimos años con población migrante y que tiene estrategias 'artesanales' de interculturalidad; o sea, no hay políticas, pero artesanalmente o de la forma que van encontrando, van incorporando, aprenden palabras en el idioma originario, traducen, hacen carteles [...]. Depende de la voluntad de cada uno, de la pregnancia de ese centro de salud, de ese servicio, de cómo es la jefatura (Laura, Grupo de Salud y Migraciones del Ministerio de Salud de CABA, comunicación personal, 28 de diciembre de 2015).

Dos cuestiones importantes se desprenden de lo anterior. Primero, si bien existen políticas de Estado con un enfoque intercultural destinadas a ampliar y mejorar la atención en SSR, éstas suelen ser insuficientes y llegan a cubrir una mínima parte de los servicios de atención. En segundo lugar, y como consecuencia de lo anterior, muchas de las estrategias alternativas al modelo biomédico tradicional desplegadas desde el sistema de salud dependen del voluntarismo de quien las aplica. En efecto, con relación a esto último, una profesional perteneciente al equipo de Parto Respetado de un Hospital Público de la CABA, explica:

Está todo comprado por nosotras. La pelota, las tiras, la red donde ponemos la pelotita de tenis y la pelota grande, donde colgamos nosotros las tiras. ADOM, que es la Asociación de Obstétricas Municipales, nos donó el banquito, porque ellas hicieron un modelo de banquito y [lo] donaron a los hospitales que trabajamos de esta forma (Adriana, obstétrica de un Hospital Público de la CABA; comunicación personal, 6 de enero de 2016).

Precisamente uno de los principales obstáculos que se presentan al intentar ampliar el alcance de las estrategias tendientes a incorporar lo cultural en el quehacer de la salud, o a flexibilizar el modelo de atención estandarizada, radica en que en gran medida dependen del voluntarismo de los profesionales y administradores de la salud. Es por ello que este tipo de iniciativas suele ser la excepción, y no la norma. En un nivel macro nos topamos con la siguiente reflexión, complementaria al carácter voluntarista analizado:

Todo [el sistema] funciona como una urgencia. Todo lo que tiene que ver con pensar en serio un seguimiento a largo plazo tiene que ver con un funcionamiento más interdisciplinario que tenga en cuenta otros aspectos que 
no sean simplemente los aspectos más biomédicos orientados a resolver la urgencia (Laura, Grupo de Salud y Migraciones del Ministerio de Salud de CABA, comunicación personal, 28 de diciembre de 2015).

Al indagar acerca de las principales resistencias que emergen en la tarea de incorporar estrategias que garanticen los derechos de las mujeres migrantes en el ámbito de la SSR y, particularmente, en el ámbito de la atención de partos, se advierte que la infraestructura aparece como un argumento "objetivo" para tomar distancia del compromiso de asegurar tales derechos:

Antes de adaptar el dilatante para este tipo de partos [partos respetados], las pacientes nuestras siempre estaban acompañadas en el dilatante. En la sala no se puede porque es una sala común y sí podían estar acompañadas, pero con mujeres, y los maridos nos quedaban afuera. [...] La ley qué dice, que la paciente tiene que estar acompañada y todo lo demás, siempre y cuando el espacio físico nos dé, y nosotros tenemos las salas de parto totalmente separadas una de la otra. Hay hospitales que no, que las separa una cortina. Entonces vos tenés al padre de una agarrando la cabeza y en frente tenés el otro y ¿estamos enfrente? (Adriana, obstétrica del Hospital Público de la CABA, comunicación personal, 6 de enero de 2016).

A partir de lo anterior cabe preguntarse en qué medida es atendible el argumento relativo a las condiciones materiales, cuando lo que entra en juego es la garantía de los derechos que protegen a las usuarias del sistema de SSR ¿dónde está el límite para determinar hasta qué punto dichas condiciones impiden el cumplimiento de un derecho básico, como el estar acompañadas durante el parto y el trabajo de parto? Al respecto, una de las informantes entrevistadas, reflexiona:

Hay lugares donde te dicen [...] que no se puede implementar lo del parto respetado porque no están dadas las condiciones edilicias. Dicen: 'bueno, primero habría que modificar las cuestiones edilicias, el cambio de lugar, porque hay un montón de mujeres en la misma sala, porque no hay intimidad, entonces no se puede hacer el parto así'. Eso se escucha mucho. Como que cualquier innovación del lado de los derechos de los usuarios es resistida con diferentes argumentos [...] Entonces ¿cuánta motivación tienen los equipos de salud para poder modificar prácticas que vayan del lado de la perspectiva de derechos de los usuarios? ¿Cuánta razón tienen? (Laura, Grupo de Salud y Migraciones del Ministerio de Salud de CABA, comunicación personal, 28 de diciembre de 2015).

Otro obstáculo que emerge en el proceso de comunicación intercultural refiere a las diferencias lingüísticas e idiomáticas. Esto es particularmente cierto cuando se trata de nombrar el cuerpo y describir sus procesos fisiológicos, aspectos profundamente atravesados por la cultura. En este sentido, una de las informantes clave advierte sobre las diferencias que aparecen en el terreno de la SSR de las migrantes limítrofes: 
A veces había formas de mencionar partes del cuerpo o enfermedades que también les costaba entender porque no era la misma forma de identificarlos en su país de origen. Por ejemplo, en la campaña hablamos de enfermedades venéreas y los equipos de salud nos querían matar. Si yo hablo de infecciones de transmisión sexual, la señora no sabe de qué estoy hablando. Si pongo el spot de enfermedades venéreas ella sí va a entender. Entonces ahí hay un desencuentro. Como que en el diálogo hay un desencuentro constante. La cuestión es cómo ayudar a que ese diálogo sea posible desde diferentes instancias (María, CAREF, comunicación personal, 14 de enero de 2016).

Finalmente, se desprende de las entrevistas que uno de los principales desafíos en materia de salud intercultural es el de superar la idea de que la salud representa una tarea individual, en vez de concebirla como un sistema de participación colectiva. Esto cobra especial relevancia cuando se trata de la SSR, cuyo foco de responsabilidad suele estar puesto en la usuaria:

Algo que se hace muchísimo es culpabilizar a las usuarias por sus padecimientos, porque vinieron tarde, porque vinieron antes. [...] La falta de lectura acerca de cuáles son las inequidades y tomar la salud como una cuestión de responsabilidad individual. [...] Los profesionales te dicen 'acá no pasa por una cuestión de migrante o no migrante, pasa por si se preocupan o no las madres por la salud de sus hijos'. Ese tono moralizante, culpabilizador y personal de responsabilizar a la mujer migrante porque no vino a las cinco de la mañana a sacarle el turno a su hijo para venir después otro día. [...] Toda esta perspectiva culpabilizadora de la familia tiende al prejuicio sobre la mujer [...] incide en que esa persona vuelva o no vuelva (Laura, Grupo de Salud y Migraciones del Ministerio de Salud de CABA, comunicación personal, 28 de diciembre de 2015).

Con relación a las percepciones de las migrantes entrevistadas provenientes de países limítrofes y del Perú, surge la pregunta ¿en qué medida estas mujeres poseen acceso efectivo a los servicios de SSR y cómo es, de acuerdo a sus experiencias, la atención recibida en los establecimientos locales de salud? Una característica común es la valoración positiva acerca del acceso y la calidad de los servicios en Argentina y, particularmente, en la CABA. Las entrevistadas destacan positivamente la gratuidad de la atención y la diversidad de cuidados preventivos que abarcan, especialmente con relación al embarazo, el parto y los cuidados del recién nacido. En contraste, los servicios de SSR en los países de origen son percibidos como precarios, de acceso deficitario, e insuficientes para dar respuestas satisfactorias a sus demandas. Asimismo, advierten que en sus contextos de origen, los temas que involucran a la sexualidad femenina se ubican en un terreno tabú. Esto último se asocia con que gran parte del transcurso de sus vidas en las sociedades emisoras, estas mujeres pudieron acceder de manera limitada e informal a información acerca de los métodos para prevenir embarazos, los cuidados para evitar infecciones de transmisión sexual o la necesidad de efectuar controles preventivos periódicos. 
Respecto de la sexualidad, yo me enteré acá que el gobierno brinda preservativos, brinda anticoncepción, además de que la salud es gratuita. Si tenés dudas con respecto a una enfermedad de transmisión sexual te lo podés hacer directamente. No hay tabú acá [...] y eso en Paraguay, no; en Paraguay no hay (Elsa, Paraguay, 24 años; comunicación personal, 12 de diciembre de 2015).

En esta misma línea, Antonia (Bolivia, 43 años) afirma:

Allá no me internaría en un hospital de Bolivia, al menos en la frontera donde estoy [Villazón], porque hay médicos, pero no son muy profesionales. En la ciudad seguramente que sí, pero en los pueblos chiquitos, no (comunicación personal, 14 de enero de 2016).

Un aspecto adicional que emana de los discursos de las entrevistadas, es la aparente relación que existe entre el tiempo de residencia en Argentina y el nivel de acceso a los servicios de SSR y la exigibilidad de los DSR que les competen. María, oriunda de Paraguay (35 años), que migró cuando estaba embarazada, relata:

Cuando recién llegué era todo nuevo. Como no tenía la precaria [documento que acredita la radicación temporaria en Argentina] no me hice los controles del embarazo [de su tercer hijo]. Solo al final del embarazo fui porque tenía mucho dolor. [...] Me trataron bien y no me pidieron documento. Yo tenía miedo de eso, 'qué pasa si me piden la precaria'. Al final estuvo todo bien. [...] Ahora [embarazo del cuarto hijo] empecé a verme desde que me enteré (comunicación personal, 6 de enero de 2016).

En general, las entrevistadas no encontraron diferencias en cuanto a la atención durante el proceso de gestación y en parto entre su país de origen y su lugar de recepción, exceptuando el hecho de que el servicio no es completamente gratis, como sí lo es en la Argentina.

Es la misma cosa, te exigen el control más por el riesgo, por si está atravesado, por si tenés que tener cesárea. En Paraguay era bastante normal tener [el parto] en la casa. Ahora menos. Ahora hay salitas y de ahí te derivan a hospital grande (Inés, 28 años, Paraguay; comunicación personal, 6 de enero de 2016).

La partera, tu mamá, o el esposo, sí hay diferencias. En el hospital, no; vas con las enfermeras o parteras. [...] Allá si te haces el tratamiento con ese médico, ese médico te atiende el parto, te va diciendo cómo va a ser el parto (Fernanda, 42 años, Perú; comunicación personal, 6 de enero de 2016).

El acceso a la información clara, completa y verdadera por parte del equipo integral de salud es uno de los principios fundamentales de la Ley Nacional N²6.529 de Derechos del Paciente y de la Ley Nacional 25.929 de Parto Respetado. Entre las garantías consignadas en esta última legislación se encuentran el derecho que tiene toda mujer a ser informada 
sobre las distintas intervenciones médicas que pueden tener lugar durante el parto y el postparto, y a participar activamente en las decisiones acerca de las distintas alternativas de intervención. Dentro del conjunto de experiencias de parto narradas por las migrantes entrevistadas, la vulneración al derecho a la información es una constante:

Entrevistadora: ¿Te hicieron episiotomía?

Paula: Sí. Estaba anestesiada, pero me di cuenta cuando me cortaron [...] no me dolió. Le pregunté a la partera cuánto me habían cortado, cuántos puntos y me dijo 'esa información nosotros acá no la damos' (Paula, 30 años, Paraguay; comunicación personal, 3 de diciembre de 2015).

En suma, y a la luz de los objetivos planteados por el PNSSyPR, las entrevistas efectuadas permiten sostener que las migrantes ven satisfechas gran parte de sus demandas en este ámbito, particularmente en lo que se refiere a los cuidados durante el embarazo y la atención de partos. Es de común acuerdo que el sistema de salud en Argentina, y más específicamente en la CABA, es capaz de dar respuestas efectivas a las necesidades que se manifiestan en el campo de la SSR. Sin embargo, al preguntarnos sobre los elementos que sustentan esta percepción, la respuesta es menos alentadora: tales apreciaciones surgen, en gran medida, de contraponer la propia experiencia en los servicios de SSR entre Argentina y el país de origen.

\section{CONCLUSIONES}

Los DSR representan un abanico amplio de garantías vinculadas directamente con la dignidad, la igualdad y la autonomía de las personas. Adoptar medidas para asegurar su ejercicio pleno a toda la población es un trabajo que apunta a profundizar y reforzar los derechos humanos. El análisis aquí presentado, relativo a las dimensiones reproductiva y no reproductiva de los DSR, constituye un esfuerzo en esta dirección, al intentar visibilizar aspectos que, o bien han recibido escasa atención en el ámbito académico y de políticas públicas -como es el caso de la fecundidad adolescente de las migrantes-, o bien suelen ser analizados desde una óptica de derechos disociada de una mirada intercultural. Los siguientes puntos representan una síntesis de los principales hallazgos y conclusiones derivados de este trabajo:

- En materia de reproducción adolescente, los colectivos migratorios analizados exhiben resultados alarmantes, que requieren de acciones oportunas por parte de los organismos del Estado, puntualmente en las áreas de salud y de protección de la infancia. Las futuras investigaciones deberían apuntar a analizar las representaciones de las adolescentes migrantes respecto de sus aspiraciones reproductivas y, por otro lado, a evaluar si los altos porcentajes de maternidad en la adolescencia dentro de las colectividades de inmigrantes responden, en realidad, a situaciones de abuso sexual. Justificar estos resultados sobre el argumento de que las migrantes "traen" un determinado nivel y calendario reproductivo de su país de origen, no es otra 
cosa que exotizar la inmigración y desconocer que tras niñas-madres se esconden adultos ultrajadores.

- Antes que la nacionalidad de origen de las migrantes, el tiempo de la migración es, aparentemente, un factor que estaría condicionando el acceso a los SSR, incluido el acceso a las pruebas de cuidados preventivos. Si bien las brechas que existen en los porcentajes de atención entre las migrantes recientes y las migrantes antiguas no son abruptas - de hecho, son más bien alentadoras-, habría que prestar atención a este primer grupo y generar estrategias que contribuyan a reducir tal diferencia.

- En la CABA existe una cifra no desdeñable de migrantes que conviven en pareja y que no utiliza MAC, pese a haber completado sus aspiraciones reproductivas. Esto podría sugerir que existe una tarea pendiente en lo que respecta a la prevención de embarazos no deseados. Es importante advertir que las campañas de planificación familiar tienden a depositar la responsabilidad de la prevención de embarazos en la mujer. A nivel social, suele incluso sobrevalorarse la píldora y la $\mathrm{T}$ de cobre por sobre otros MAC para la prevención de embarazos. En tal sentido, las campañas en este ámbito debiesen reforzar la vasectomía y el uso condón masculino como métodos alternativos de planificación.

- Con relación a la incorporación de la dimensión intercultural en el ámbito de la SSR, se advierte que dentro del amplio entramado de profesionales y administradores que conforman el sistema sanitario, se han logrado implementar "estrategias artesanales de interculturalidad", que en sí mismas permiten reforzar la promoción y protección de determinados DSR de las migrantes y de la población en general. Si bien la normativa vigente incorpora y tiende a reforzar este lineamiento, al tratarse de estrategias artesanales se concluye que aún persiste una brecha entre las leyes existentes y su implementación en el sistema de salud. Asimismo, todavía persisten viejas resistencias y obstáculos que limitan la comprensión de lo cultural como un aspecto constitutivo de la persona migrante. Queda por resolver ahora la pregunta de acerca de qué oportunidades reales tienen los profesionales y administradores de la salud de pensar lo cultural como una dimensión subjetiva amplia, y qué otras alternativas existen, además de las estrategias artesanales descritas, para corporizarlo.

- El análisis expuesto acerca del estado de los DSR de las migrantes de países limítrofes que residen en la CABA debe ser considerado una aproximación exploratoria y acotada a esta temática. Algunas alternativas de investigaciones ulteriores que podrían contribuir a examinar en mayor detalle este panorama son la aplicabilidad de la ley $\mathrm{N}^{\circ} 25.929$ de Parto y Nacimiento 
Humanizado desde la voz de las propias migrantes, el fenómeno del aborto inducido en las colectividades de migrantes, actualización sobre la trata de migrantes con fines de explotación sexual, la situación de los DSR de la población LGBTI, entre otros.

\section{REFERENCIAS}

Agu, J., Lobo, R., Crawford, G. y Chigwada, B. (2016). Migrant Sexual Health HelpSeeking and Experiences of Stigmatization and Discrimination in Perth, Western Australia: Exploring Barriers and Enablers. International Journal of Environmental Research and Public Health, 13(5), 1-22. Recuperado de https://www.mdpi.com/16604601/13/5/485/htm; https://doi.org/10.3390/ijerph13050485

Aizenberg, L. y Maure, G. (2017). Migración, salud y género: abordajes de proveedores de salud en la atención de mujeres migrantes bolivianas en la provincia de Mendoza, Argentina, REMHU: Revista Interdisciplinar da Mobilidade Humana, 25(51), 149-164.

Carballo, M. (2006). Female Migrants, Reproductive Health, HIV/AIDS and the Rights of Women, UNFPA-IOM. Recuperado de http://legeforeningen.no/Fagmed/Norskgynekologisk-forening/Nyheter/20061/female-migrants-reproductive-health-hivaidsthe-rights-of-women/

Cerrutti, M. (2009). Diagnóstico de las poblaciones de inmigrantes en la Argentina, Serie de Documentos de la Dirección Nacional de Población, 2. Buenos Aires: Dirección Nacional de Población.

Cerrutti, M. (2010). Salud y migración internacional: mujeres bolivianas en la Argentina. Buenos Aires: Fondo de Población de las Naciones Unidas/ Centro de Estudios de Población.

Chen, M., Rosero, L., Brenes, G., León, M., González, M. I. y Vanegas, J. C. (2001). Salud reproductiva y migración nicaragüense en Costa Rica 1999-2000: Resultados de una Encuesta Nacional de Salud Reproductiva. San José, Costa Rica: Universidad de Costa Rica.

Chiavarini, M., Lanari, D., Minelli, L., Pieroni, L. y Salmasi, L. (2016). Immigrant mothers and access to prenatal care: evidence from a regional population study in Italy. British Medical Journal open, 6(2), 1-9.

Comelles, J. (2004). El regreso de las culturas. Diversidad y práctica médica en el S. XXI. En G. Fernández Juárez (Coord.), Salud e interculturalidad en América Latina. Perspectivas antropológicas, (pp. 6-21). Quito: Abya Yala. 
Dirección Nacional de Población. (2011). Encuesta sobre Migración, Fecundidad y Familia 2011. Recuperado de https://argentina.unfpa.org/es/publicaciones/encuestamigraci\%C3\%B3n-familia-y-fecundidad-emff

Fernández Juárez, G. (2011). Claroscuros de la salud intercultural en América Latina. Nuevo Mundo Mundos Nuevos. Débats, (s/n). Recuperado de http://journals.openedition.org/nuevomundo/61188. doi.org/10.4000/nuevomundo.61188

Haour-Knipe, M. y Grondin, D. (2003). Sexual health of mobile and migrant populations. Sexual Health Exchanges, 2, 1-3.

Heaman, M., Bayrampour, H., Kingston, D., Blondel, B., Gissler, M., Roth, C. y Gagnon, A. (2013). Migrant women's utilization of prenatal care: a systematic review. Maternal and Child Health Journal, 17(5), 816-836.

Infante, C., Silván, R., Caballero, M. y Campero, L. (2013). Sexualidad del migrante: experiencias y derechos sexuales de centroamericanos en tránsito a los Estados Unidos. Salud pública Méx, 55(1), 58-64.

International Agency for Research on Cancer (IARC). (2012). Base de datos IARC53. Recuperado de http://p53.iarc.fr/TP53GeneVariations.aspx

Instituto Nacional de Estadística y Censos (INDEC). (2015). Censo Nacional de Población, Hogares y $2010 . \quad$ Viviendas Recuperado de https://redatam.indec.gob.ar/argbin/RpWebEngine.exe/PortalAction?\&MODE=MAIN\& BASE $=$ CPV2010A\&MAIN=WebServerMain.inl

Jelin, E., Grimson, A. y Zamberlin, N. (2006). Los pacientes extranjeros en la mira. En E. Jelin (Ed.), Salud y migración regional. Ciudadania, discriminación y comunicación intercultural (pp. 47-56). Buenos Aires: Instituto de Desarrollo Económico y Social.

Magliano, M. J. (2015). Interseccionalidad y migraciones: potencialidades y desafíos. Revista Estudos Feministas, 23(3), 691-712.

Monte, M. E. y Gavernet, L. (2012). La incorporación de los derechos sexuales y reproductivos en las constituciones de Argentina, Venezuela, Ecuador y Bolivia. En J. M. Morán Faúndes, M. C. Sgró Ruatay y J. M. Vaggione (Eds.), Sexualidades, desigualdades $y$ derechos. Reflexiones en torno a los derechos sexuales y reproductivos, (pp. 157-189). Córdoba, Argentina: Ciencia, Derecho y Sociedad.

Organización de las Naciones Unidas. (2016). Situación de los migrantes en tránsito. Informe de la Oficina del Alto Comisionado de las Naciones Unidas para los Derechos Humanos, Informe de la Oficina del Alto Comisionado de las Naciones Unidas para los Derechos Humanos, 31er período de sesiones. Recuperado de https://www.acnur.org/fileadmin/Documentos/BDL/2016/10356.pdf 
Organización de las Naciones Unidas. (1995). Informe de la Conferencia Internacional sobre la Población y el Desarrollo, El Cairo, 5-13 septiembre 1994. Recuperado de https://www.unfpa.org/sites/default/files/pub-pdf/icpd_spa.pdf

Programa Conjunto de las Naciones Unidas sobre el VIH/Sida (ONUSIDA). (2013). Global report: UNAIDS report on the global AIDS epidemic 2013. Recuperado de https://www.unaids.org/sites/default/files/media_asset/UNAIDS_Global_Report_2013 en_1.pdf

Organización Mundial de la Salud (OMS). (2009). Women and health: today's evidence tomorrow's agenda. Ginebra: Organización Mundial de la Salud.

Programa de las Naciones Unidas para el Desarrolla (PNUD). (2008). HIV vulnerabilities faced by women migrants: from Asia to the Arab States. Colombo: Programa de las Naciones Unidas para el Desarrollo.

Sedgh, G., Bearak, J., Singh, S., Bankole, A., Popinchalk, A., Ganatra, B., [...] Alkema, L. (2016). Abortion incidence between 1990 and 2014: global, regional, and subregional levels and trends. The Lancet, 388(10041), 258-267.

Smith, A. y LeVoy, M. (2016). Los derechos sexuales y reproductivos de los migrantes en situación administrativa irregular cerrando la brecha entre sus derechos y la realidad en la Unión Europea. Recuperado de http://picum.org/wpcontent/uploads/2017/11/Sexual-and-Reproductive-Health-Rights_ES.pdf

Wang, L. (2010). Ideas y prácticas en torno a la Salud Sexual y Reproductiva en jóvenes migrantes paraguayas que se trasladan a la Argentina. Eä Revista de Humanidades Médicas \& Estudios Sociales de la Ciencia y la Tecnología, 2(2),65-94.

Zamberlin, N., Thouyaret, L. y Arrossi, S. (2011). Lo que piensan las mujeres: conocimientos y percepciones sobre cáncer de cuello de útero y realización de PAP. Buenos Aires: Ministerio de Salud de la Nación/UNICEF/Organización Panamericana de la Salud. 